\title{
Detection of bone marrow metastases in neuroblastoma using a short term tissue culture technique
}

\author{
J A Adams, A M Kelsey, T F Carr, R F Stevens, P Morris-Jones
}

\begin{abstract}
Aims: To improve detection of neuroblastoma metastases in the bone marrow: morphological evaluation of bone marrow is a routine and important component of the clinical staging of neuroblastoma and it depends on the successful identification of tumour cells which may only be present at extremely low levels.

Methods: Bone marrow mononuclear cells from patients with neuroblastoma were incubated in a simple suspension culture and examined regularly using an inverted objective microscope. In some cases cytospins of the cultured cells were examined further using morphological and immunocytological techniques.

Results: In some cultures spheroidal clumps of cells could be seen growing after only a few days. If the marrow was cultured for a longer period these spheroids continued to increase in size, became adherent to the stromal cell layer on the culture flask floor, and put out long characteristic processes (neurites). Morphological and immunocytological examination of cytospins from these cultures confirmed these cells as neuroblasts.

Conclusions: This method has provided the sole evidence of marrow metastases in several newly diagnosed cases of neuroblastoma in which the bone marrow had shown no evidence of tumour using standard morphological and fluorescent immunocytological techniques. Although negative cultures do not preclude the presence of neuroblastoma, this method is a useful adjunct to the standard techniques.
\end{abstract}

Comparison of trephine biopsy, immunofluorescence and tissue culture for the detection of neuroblastoma cells infiltrating bone marrow

\begin{tabular}{lllll}
\hline \multirow{2}{*}{ Patient } & & \multicolumn{2}{l}{ Neuroblastoma detected by } \\
\cline { 3 - 5 } & Treated & Trephine & Immunofluorescence & Tissue culture \\
\hline 1 & No & ND & No & Yes \\
2 & Yes & No & No & Yes \\
3 & No & No & No & Yes \\
4 & No & ND & Yes & Yes \\
& No & Yes & No & No \\
5 & Yes & No & ND & Yes \\
& No & Yes & No & No \\
6 & Yes & Yes & No & No \\
7 & Yes & No & No & No \\
\hline
\end{tabular}

ND, no data.
Detection of bone marrow infiltration is of clinical importance in the management of children with neuroblastoma for several reasons. Bone marrow must be examined: (i) at diagnosis to determine the stage of the disease and thus treatment and prognosis; (ii) during treatment to assess response; (iii) before any marrow is harvested for potential autologous rescue; and (iv) after the completion of treatment to confirm or refute suspected relapse. ${ }^{12}$ Bone marrow infiltration in disseminated neuroblastoma can, however, be difficult to detect. Trephine biopsies are thought to be more effective than aspirates and multiple site sampling improves tumour detection rate over that obtained with single site sampling. ${ }^{34}$ Standard cytological analysis of bone marrow aspirates and histological analysis of trephine biopsies however depend on the morphological identification of tumour cells which may only be present in small numbers. Immunostaining using specific monoclonal antibodies which recognise antigens carried by the neuroblasts can improve detection of bone marrow invasion. ${ }^{25}$ Unfortunately two of the most promising monoclonal antibodies against neuroectodermal tissue, UJ13A and UJ127 11 , are of no value in the study of formalin fixed tissue. Other studies indicate that immunostaining has a limited role in the detection of metastatic neuroblastoma ${ }^{67}$ although Caillaud et al found staining for neurone specific enolase in paraffin wax embedded trephine material to be a sensitive immunomarker for detection of marrow invasion. ${ }^{8}$

We applied a short-term tissue culture technique to a small series of bone marrow aspirate samples from children with neuroblastoma. The findings from bone marrow cultures were compared to those from histological and immunofluorescence studies performed on the same marrow samples.

\section{Methods}

Bone marrow samples from seven patients were studied. Five were newly diagnosed patients and three of these were further studied after their first course of treatment.

Bone marrow trephine biopsy samples were processed for routine histopathological examination and in some cases the biopsy samples were immunostained using a monoclonal antibody to neurone specific enolase (Dako Ltd). Bone marrow aspirates were 


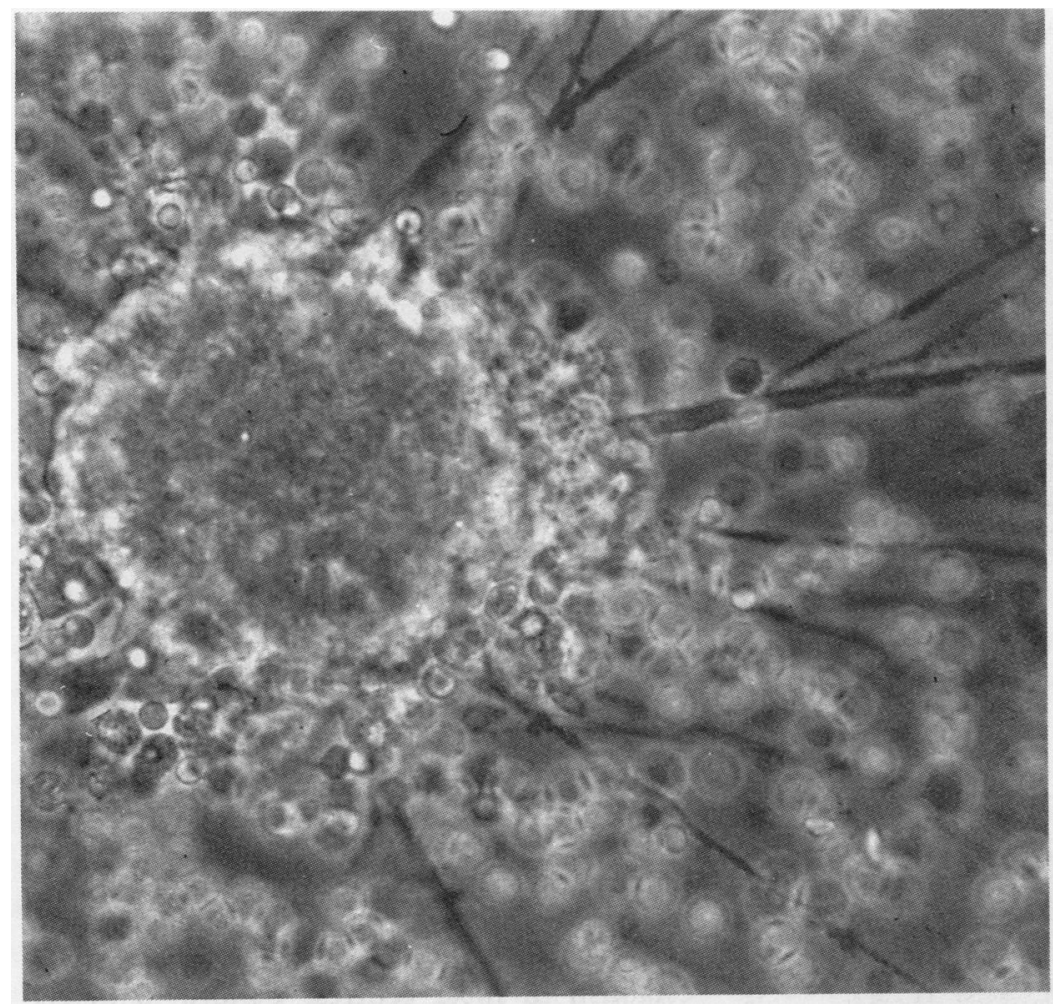

Figure 1 Neuroblastoma cells growing in tissue culture after 14 days.

anticoagulated with $10 \mathrm{IU} / \mathrm{ml}$ preservative free heparin and divided into two samples. One sample was examined for contamination with neuroblasts by indirect immunofluorescence using the following monoclonal antibodies: $\mathrm{UJ} 13 \mathrm{~A}$, and a mixture of UJ181.4, UJ127.11, and $5 \cdot 1 \cdot \mathrm{H} 11$. These monoclonal antibodies detect antigens present on cells of neuroectodermal origin and were kindly supplied by Dr J Kemshead. Samples were analysed using a fluorescence activated cell analyser (Coulter

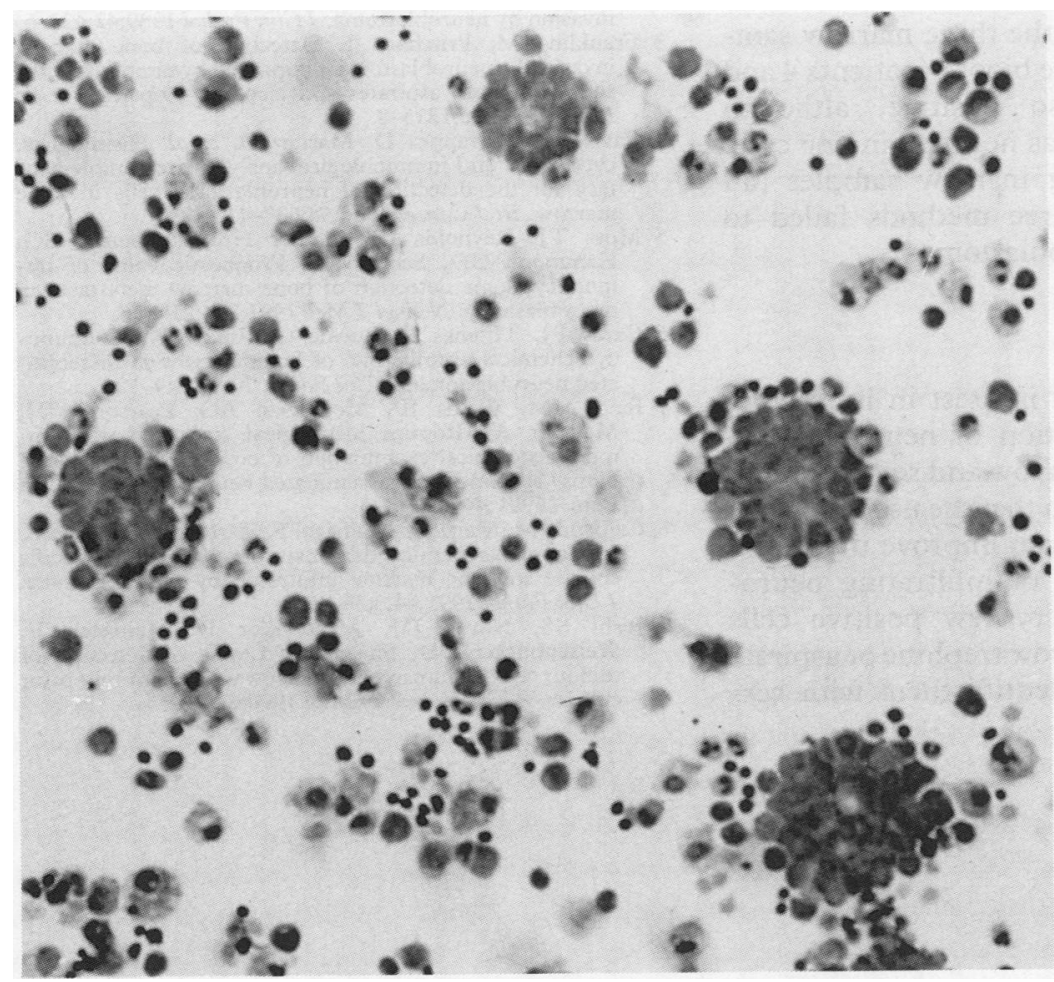

Figure 2 Cytospin of neuroblastoma cells from a culture at day 5 stained with Giemsa.
EPICS). The other bone marrow aspirate sample was used for tissue culture. The bone marrow mononuclear cell fraction was isolated by density centrifugation on Ficoll-Hypaque (Lymphoprep, Nyegaard, UK), washed once, and resuspended at a concentration of $0.5-1 \times$ $10^{6} \mathrm{cells} / \mathrm{ml}$ in McCoy's $5 \mathrm{~A}$ tissue culture medium supplemented with $15 \%$ heat inactivated fetal bovine serum (Gibco BRL). Aliquots of the cell suspension $(5 \mathrm{ml})$ were incubated in $25 \mathrm{~cm}^{2}$ tissue culture flasks (Costar Ltd) in $5 \% \mathrm{CO}_{2}$ in air at $37^{\circ} \mathrm{C}$. Cultures were examined at initiation and at regular intervals up to 14 days of culture using an inverted objective microscope (Olympus) for formation of clumps of neuroblasts. These cells were initially seen as non-adherent spheroids of cells but later settled on the bone marrow stromal layer forming on the flask floor. In some cases these neuroblast spheroids put out characteristic processes or neurites. In order to confirm that these cells were neuroblasts, samples from the flasks were cytospun onto slides and immunostaining performed. A panel of antibodies: UJ13A, a mixture of UJ181.4 plus UJ127.11 plus $5 \cdot 1 \cdot \mathrm{H} 11$, anti-neurofilament (Dako Ltd) and anti-neurone specific enolase (Dako Ltd) were used in an APAAP technique.

\section{Results}

The detection of marrow infiltration by neuroblastoma using three different techniques, trephine biopsy, immunofluorescence, and tissue culture is shown in the table. Of the five newly diagnosed patients studied (patients 1-5) three showed no marrow infiltration with either the trephine or immunofluorescence techniques (patients 1-3), as did one of these patients after treatment (patient 4). No trephine biopsy was performed on patients 1 and 3 at diagnosis as they were both less than six weeks old and patient 3 was staged as $4 S$, the native marrow smears were not diagnostic of neuroblastoma. After a short period in tissue culture clumps of neuroblasts could be detected in the marrows of patients $1-3$ using the inverted microscope. These spheroids appeared to be derived from single dispersed neuroblasts as no clumps were seen in the newly initiated cultures. The time to formation of noticeable spheroids of neuroblasts in these three patients was from three to eight days and the clumps continued to increase in size for several days after their initial appearance. The presence of neuroblasts was confirmed by morphological examination and/or immunostaining of cytospins made from the cultured bone marrow cells. Dividing cells of other lineages were not found to form spheroids in these cultures. Figure 1 shows neuroblastoma cells growing in a bone marrow culture after 14 days and the production of the characteristic processes or neurites. Figure 2 shows cells from a culture at day 5 which have been cytospun and stained with Giemsa. Clumps of cells morphologically resembling neuroblasts can be seen clearly. Figure 3 shows a spheroid stained with Giemsa and fig 4 a spheroid showing positive immunostaining with $\mathrm{UJ} 13 \mathrm{~A}$ in an 
Figure 3 Spheroid of neuroblastoma cells from a culture at day 8 stained with Giemsa.
Figure 4 Spheroid showing positive immunostaining with $U J 13 A$ in an $A P A A P$ technique.

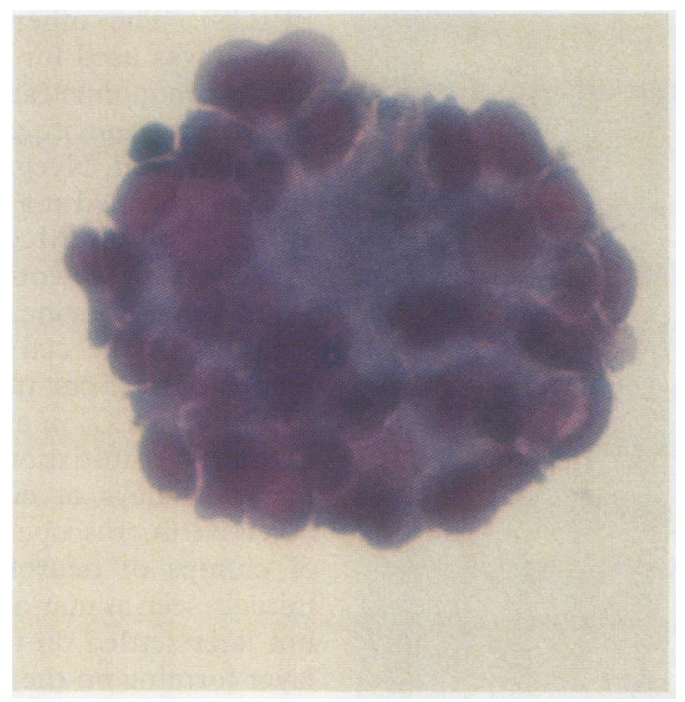

tainty. Using this short term tissue culture method we have been able to increase the number of neuroblasts in marrow aspirates from newly diagnosed patients thus making detection using either morphology or specific immunostaining more reliable. The time taken to form and the number of neuroblast spheroids depends on the initial number of infiltrating neuroblasts and their growth potential. We believe that short term tissue culture can be a valuable adjunct to the standard range of techniques, and other workers have also found that culture techniques can increase the sensitivity of detection of occult tumour cells in human bone marrow. ${ }^{9}$ Tissue culture is not only useful with diagnostic marrow specimens but also has applications in the assessment of bone marrow used for autografting and the efficiency of the various techniques used in attempts to purge bone marrow of infiltrating tumour cells. This method can also be adapted to permit further studies on the growth characteristics of neuroblastoma in the bone marrow. Short term tissue culture is a simple, cheap technique requiring relatively little expertise and specialist equipment and should be easy to establish in most centres. Failure to detect neuroblastoma infiltrating the marrow does not preclude its presence but by using this method we can increase the detection rate of very low numbers of infiltrating neuroblastoma cells.

JAA was supported by a grant from the Royal Manchester Children's Hospital Endowment Fund and the work was performed in the Giving for Living Research Foundation Laboratories, Royal Manchester Children's Hospital.

1 Brodeur GM, Seegar RC, Barrett A, et al. International criteria for diagnosis, staging and response to treatment in patients with neuroblastoma. J Clin Oncol 1988;6: patients

2 Rogers DW, Treleaven JG, Kemshead JT, Pritchard J. Monoclonal antibodies for detecting bone marrow invasion by neuroblastoma. J Clin Pathol 1989;42:422-6.

APAAP technique. In the three marrow samples positive on trephine biopsy (patients 4 and 5) cultures were also positive, although immunofluorescence was negative in one case. In the four remaining marrow samples (all post-treatment), all three methods failed to detect infiltrating neuroblastoma.

\section{Discussion}

There is much current interest in improving the methods for detection of neuroblastoma infiltrating the bone marrow and several recent publications have shown that the use of specific monoclonal antibodies can improve the detection of small numbers of infiltrating neuroblasts. If there are very few positive cells present in the bone marrow trephine or aspirate it can be difficult to identify them with cer- 